\title{
ON SOME FIXED POINT THEOREMS FOR MAPPINGS SATISFYING A NEW TYPE OF IMPLICIT RELATION
}

\section{Valeriu Popa}

\begin{abstract}
In this paper we introduce a new class of functions $F: R_{+}^{6} \rightarrow R$ such that the fulfilment of the inequality of type (3) for $x, y$ in $X$, ensures the existence and the uniqueness of a fixed point.
\end{abstract}

\section{Introduction}

The notion of contractive mapping has been introduced by Banach in [1].

In the last thirty years different types of generalizations of this concept appeared. The connection between them have been studied in different papers, for example [2], [3], [5]-[9].

Let $(X, d)$ be a metric space and $T:(X, d) \rightarrow(X, d)$ a mapping in essence, $T$ is a generalized contraction if an inequality of type

$$
d(T x, T y) \leq f(d(x, y), d(x, T x), d(y, T y), d(x, T y), d(y, T x))
$$

holds for $x, y \in X$, where $f: R_{+}^{5} \rightarrow R$ satisfies some properties or has a special form.

In [4], the present author established a class of mappings $F: R_{+}^{6} \rightarrow R$ such as the fulfilment of the inequality of type

$$
F(d(T x, T y), d(x, y), d(x, T x), d(y, T y), d(x, T y), d(y, T x)) \leq 0
$$

for $x, y \in X$, ensures the existence and the uniqueness of a fixed point for $T$.

The purpose of this paper is to introduce a new class of mappings $F: R_{+}^{6} \rightarrow R$ such that the fulfilment of the inequality of type

$$
F\left(d(T x, T y), d(x, y), d(x, T y), d(y, T y), d\left(y, T^{2} x\right), d(y, T x)\right) \leq 0
$$

for $x, y \in X$, ensures the existence and the uniqueness of a fixed point for $T$.

AMS (MOS) Subject Classification 1991. Primary: 54H25

Key words and phrases: Fixed point, complete metric space, compact metric space, implicit relation. 


\section{Implicit relations}

Let $\Phi$ be the set of all real continuous functions $F\left(t_{1}, \ldots, t_{6}\right): R_{+}^{6} \rightarrow R$ satisfying the following conditions:

$\emptyset_{1}: \mathrm{F}$ is nonincreasing in variable $t_{5}$,

$\emptyset_{h}$ : there exists $h \in[0,1)$ such that for every $u, v \geq 0$

$F(u, v, v, u, u, 0) \leq 0$ implies $u \leq h v$;

$\emptyset_{u}: F(u, u, 0,0, u, u)>0, \forall u>0$.

Ex.1. $F\left(t_{1}, \ldots, t_{6}\right)=t_{1}^{2}-a t_{5} t_{6}-b \max \left\{t_{2}^{2}, t_{3}^{2}, t_{4}^{2}\right\}$, where $a>0, b \geq 0$ and $a+b<1$.

$\left(\emptyset_{1}\right)$ : Obviously.

$\left(\emptyset_{h}\right)$ : Let $u>0, v \geq 0$ and $F(u, v, v, u, u, 0)=u^{2}-\operatorname{bmax}\left\{u^{2}, v^{2}\right\} \leq 0$. If $u \geq v$ then $u^{2}(1-b)<0$, a contradiction. Thus $u<v$ and $u \leq h v$, where $h=\sqrt{b}<1$.

If $u=0$ and $v \geq 0$ then $u \leq h v$.

$\left(\emptyset_{u}\right) F(u, u, 0,0, u, u)=u^{2}(1-a-b)>0, \forall u>0$.

Ex.2. $F\left(t_{1}, \ldots, t_{6}\right)=t_{1}^{2}-a t_{5} t_{6}-t_{1}\left(b t_{2}+c t_{3}+d t_{4}\right)$, where $a, b, c, d \geq 0$ and $a+b+c+d<1$.

$\left(\emptyset_{1}\right)$ : Obviously.

$\left(\emptyset_{h}\right)$ : Let $u>0, v \geq 0$ and $F(u, v, v, u, u, 0)=u^{2}-u(b v+c v+d u) \leq 0$. Then $u \leq h v$, where $h=c+b / 1-d<1$. If $u=0, v \geq 0$ then $u \leq h v$.

$\left(\emptyset_{u}\right): F(u, u, 0,0, u, u)=u^{2}(1-a-b)>0, \forall u>0$.

Ex.3. $F\left(t_{1}, \ldots, t_{6}\right)=t_{1}^{3}-a t_{1}^{2} t_{2}-b t_{1} t_{2}^{2}-c t_{2} t_{3} t_{4}-d t_{5}^{2} t_{6}$, where $a>$ $0, b, c, d \geq 0$ and $a+b+c+d<1$.

$\left(\emptyset_{1}\right)$ : Obviously.

$\left(\emptyset_{2}\right)$ : Let $u>0, v \geq 0$ and $F(u, v, v, u, u, 0)=u^{3}-a u^{2} v-b u v^{2}-c u v^{2} \leq$ 0 , which implies $u^{2}-a u v-(b+c) v^{2} \leq 0$. If $b=c=0$, then $u \leq h v$, where $0<h=a<1$.

If $b+c>0$ then $f(t)=(b+c) t^{2}+a t-1 \geq 0$, where $t=v / u>0$. Since $f(1)=(a+b+c)-1<0$, let $r>1$ be the root of equation $f(t)=0$. Then $f(t)>0$ for $t>r$ which implies $u \leq h v$, where $h=1 / r<1$. If $u=0$ then $u \leq h v$.

$$
\left(\emptyset_{u}\right): F(u, u, 0,0, u, u)=u^{3}(1-a-b-d)>0, \forall u>0 .
$$

\section{Fixed points in complete metric spaces}

Theorem 1. Let $(X, d)$ be a metric space and $T:(X, d) \rightarrow(X, d)$ be a mapping satisfying the inequality (3) for every $x, y \in X$, where $F$ satisfies condition $\left(\emptyset_{u}\right)$. Then $T$ has at most one fixed point. 
Proof. Suppose that $T$ has two fixed points $u$ and $v$ with $u \neq v$. Then by (3) we have successively

$$
\begin{gathered}
F\left(d(T u, T v), d(u, v), d(u, T u), d(v, T v), d\left(v, T^{2} u\right), d(v, T u)\right) \leq 0 \\
F(d(u, v), d(u, v), 0,0, d(u, v), d(u, v)) \leq 0
\end{gathered}
$$

a contradiction of $\left(\emptyset_{u}\right)$.

Theorem 2. Let $(X, d)$ be a metric space and $T:(X, d) \rightarrow(X, d)$ be a mapping such that there exists $h \in[0,1)$ with $d\left(T^{2} x, T x\right) \leq h d(x, T x)$ for every $x \in X$. Then for every $x \in X$ the sequence $\left\{T^{n} x\right\}$ is a Cauchy sequence.

Proof. Let $x \in X$ and the sequence $\left\{T^{n} x\right\}$. Since $d\left(T^{2} x, T x\right) \leq$ $h d(x, T x)$ by induction we have $d\left(T^{n+1} x, T^{n} x\right) \leq h^{n} d(x, T x)$. By a routine calculation it follows that $\left\{T^{n} x\right\}$ is a Cauchy sequence.

Theorem 3. Let $(X, d)$ be a complete metric space and $T:(X, d) \rightarrow$ $(X, d)$ a mapping satisfying the inequality (3) for every $x, y \in X$ where $F \in \Phi$. Then $T$ has a unique fixed point.

Proof. Let $x$ be arbitrary in $X$. We shall show that the sequence defined by $x_{n+1}=T x_{n}$ is a Cauchy sequence. From (3) for $y=T x$ we have

$$
F\left(d\left(T x, T^{2} x\right), d(x, T x), d(x, T x), d\left(T x, T^{2} x\right), d\left(T x, T^{2} x\right), 0\right) \leq 0 .
$$

By $\left(\emptyset_{h}\right)$ we have $d\left(T^{2} x, T x\right) \leq h \cdot d(x, T x)$. By Theorem $2, x_{n+1}=T^{n} x$ is a Cauchy sequence. Since $(X, d)$ is complete, there exists $u \in X$ such that $\lim x_{n}=u$.

By (3) we have successively

$$
\begin{gathered}
F\left(d\left(T x_{n}, T u\right), d\left(x_{n}, u\right), d\left(x_{n}, T x_{n}\right), d(u, T u), d\left(u, T^{2} x_{n}\right), d\left(u, T x_{n}\right)\right) \leq 0 . \\
F\left(d\left(x_{n+1}, T u\right), d\left(x_{n}, u\right), d\left(x_{n}, x_{n+1}\right), d(u, T u), d\left(u, x_{n+2}\right), d\left(u, x_{n+1}\right)\right) \leq 0 .
\end{gathered}
$$

Letting $n$ tend to infinity we have successively

$$
\begin{gathered}
F(d(u, T u), 0,0, d(u, T u), 0,0) \leq 0, \\
F(d(u, T u), 0,0, d(u, T u), d(u, T u), 0) \leq 0,
\end{gathered}
$$

which implies by $\left(\emptyset_{h}\right)$ that $u=T u$. By Theorem $1 u$ is the unique fixed point of $T$.

Corollary 1. Let $(X, d)$ be a complete metric space and $T:(X, d) \rightarrow$ $(X, d)$ satisfying one of the following inequalities:

(1.1) $d^{2}(T x, T y) \leq a d\left(y, T^{2} x\right) d(y, T x)+b \max \left\{d^{2}(x, y), d^{2}(x, T x), d^{2}(y, T y)\right\}$, 
where $a>0, b \geq 0$ and $a+b<1$, or

$$
\begin{gathered}
d^{2}(T x, T y) \leq \\
a d\left(y, T^{2} x\right) d(y, T x)+d(T x, T y)(b d(x, y)+c d(x, T x)+d d(y, T y),
\end{gathered}
$$

where $a, b, c, d \geq 0$ and $a+b+c+d<1$, or

$$
\begin{aligned}
& d^{3}(T x, T y)-a d^{2}(T x, T y) d(x, y)-b d(T x, T y) d^{2}(x, y)- \\
& \quad c d(x, y) d(x, T x) d(y, T y)-d \cdot d^{2}\left(y, T^{2} x\right) \cdot d(y, T x) \leq 0,
\end{aligned}
$$

where $a>0, b, c, d \geq 0$ and $a+b+c+d<1$ for all $x, y$ in $X$, then $T$ has unique fixed point.

Remark 1. Let $\Psi$ be the set of all real continuous functions $F\left(t_{1}, \ldots, t_{6}\right)$ : $R_{+}^{6} \rightarrow R$ satisfying the following conditions:

$\left(\Psi_{1}\right): F$ is nonincreasing in variable $t_{5}$,

$\left(\Psi_{h}\right)$ : there exists $h \in[0,1)$ such that for every $u, v \geq 0, F(u, v, u, v, u, 0) \leq$ 0 implies $u \leq h \cdot v$,

$\left(\Psi_{u}\right): F(u, u, 0,0, u, u)>0, \forall u>0$.

Theorem 4. If the inequality

$$
F\left(d(T x, T y), d(x, y), d(x, T x), d(y, T y), d\left(x, T^{2} y\right), d(x, T y)\right) \leq 0
$$

holds for all $x, y$ in $X$, where $F \in \Psi$, then $F$ has a unique fixed point.

Proof. The proof is similar to the proof of Theorem 3.

\section{Fixed points in compact metric spaces}

Let $\bar{\Phi}$ be the set of all real continuous functions $F\left(t_{1}, \ldots, t_{6}\right): R_{+}^{6} \rightarrow R$ satisfying the following conditions:

$\left(\overline{\Phi_{h}}\right)$ : For every $u \geq 0, v>0, F(u, v, v, u, u, 0)<0$ implies $u<v$, $\left(\overline{\Phi_{u}}\right): F(u, u, 0,0, u, u)>0, \forall u>0$.

Remark 2. The functions $F$ from Ex. 1-3 satisfies conditions $\left(\overline{\Phi_{h}}\right)$ and $\left(\overline{\Phi_{u}}\right)$.

Remark 3. There exists functions $F \in \bar{\Phi}$ which is increasing in variable $t_{5}$.

Ex.4. $F\left(t_{1}, \ldots, t_{6}\right)=t_{1}^{3}-c \frac{t_{2} t_{3} t_{4}}{1+t_{5}+t_{6}}$, where $0<c<1$.

$\left(\overline{\Phi_{h}}\right)$ : Let $u, v>0$ and $F(u, v, v, u, u, 0)=u^{3}-c \frac{v^{2} u}{1+u}<0$, then $u^{2}<$ $\frac{c}{1+v} v^{2}$ which implies $u<v$. If $u=0$, then $u<v$.

$\left(\overline{\Phi_{u}}\right): F(u, u, 0,0, u, u)=u^{3}>0, \forall u>0$. 
Theorem 5. Let $T$ be a continuous mapping of the compact metric space $(X, d)$ into itself such that

$$
F\left(d(T x, T y), d(x, y), d(x, T x), d(y, T y), d\left(y, T^{2} x\right), d(y, T x)\right)<0
$$

for every $x \neq y$ in $X$, where $F \in \bar{\Phi}$. Then $T$ has a unique fixed point.

Proof. Let $f(x)=d(x, T x)$ for all $x \in X$. Since $T$ is continuous, there exists a point $z \in X$ such that $f(z)=\inf \{f(x): x \in X\}$. Suppose that $z \neq T z$.

By (5) for $x=z$ and $y=T z$ we obtain

$$
F\left(d\left(T z, T^{2} z\right), d(z, T z), d(z, T z), d\left(T z, T^{2} z\right), d\left(T z, T^{2} z\right), 0\right)<0
$$

which implies $d\left(T z, T^{2} z\right)<d(z, T z)=\inf \{d(x, T x): x \in X\}$. A contradiction.

Hence, $z=T z$. From Theorem $1 z$ is the unique fixed point of $T$.

Corollary 2. Let $T$ be a continuous mapping of the compact metric space $(X, d)$ into itself such that

$$
d^{3}(T x, T y)<c \frac{d(x, y) d(x, T x) d(y, T y)}{1+d\left(y, T^{2} y\right)+d(y, T x)}
$$

for all $x \neq y$ in $X$ and $0<c<1$. Then $T$ has a unique fixed point.

Proof. The proof follows from Theorem 5 and Ex.4.

Remark 4. A Corollary analogous to Corollary 1 is obtained by Ex.13.

Remark 5. A Theorem similar to Theorem 4 is obtained for compact metric space.

\section{References}

[1] S. Banach: Sur les opérations dans les ensembles abstraits et leur application aux équations intégrales, Fund. Math., 3(1922), 133-181.

[2] J.Kincses and V.Totik: Theorems and counter-examples on contractive mappings, Math.Balcanica, 4(1990), 69-90.

[3] S.Park: On general contractive type conditions, J.Korean Math.Soc. 17(1980), 131-140.

[4] V.Popa: Fixed point theorems for implicit contractive mappings, Stud. Cerc.St., ser.Matem., 7 (1997), 129-133.

[5] B.E.Rhoades: A comparision of various definitions of contractive mappings, Trans.Amer.Math.Soc., 226(1971), 257-290. 
[6] B.E.Rhoades: A collections of contractive definitions, Math.Semin.Notes, 7(1979), 229-235.

[7] B. E. Rhoades: Contractive definitions revisited, Topological Methods in NonLinear Analysis, Contemporary Mathematics, AMS 21(1983), 189-205.

[8] B.E.Rhoades: Contractive definitions, Non Linear Analysis (Ed. T. M. Rassias), World Scientific Publishing Company, New Jersey (1988) 513-526.

[9] M. R. Tasković: Some new principles in fixed point theory, Math. Japonica, 35(1990) 645-666.

Department of Mathematics University of Bacau 5500 Bacau, Romania e-mail:vpopa@ub.ro 